\title{
UN ACERCAMIENTO A LA GESTIÓN DE CONOCIMIENTO EN EL PROCESO DE TRANSFERENCIA UNIVERSIDAD - EMPRESA
}

\author{
AN APPROACH TO KNOWLEDGE MANAGEMENT AND INNOVATION IN THE \\ CONTEXT OF THE UNIVERSITY - INDUSTRY RELATIONSHIP
}

\section{ALFREDO ESCALA CORNEJO}

Universidad Católica de Santiago de Guayaquil, Ecuador. alfredo.escala@cu.ucsg.edu.ec

RESUMEN

El estudio tuvo como objetivo evaluar la percepción de la actual gestión del conocimiento (GC) entre universidad - empresa desde la perspectiva de las autoridades académicas y directivos de empresas públicas y privadas ecuatorianas. La metodología tuvo un enfoque cuantitativo y siguió un diseño transversal, se utilizó un instrumento validado con tres dimensiones de gestión del conocimiento: creación, almacenamiento y transferencia, y aplicación y uso del conocimiento. Los resultados revelaron diferencias importantes en la percepción de las distintas actividades de la GC entre las autoridades académicas y los directivos empresariales. El estadístico $\mathrm{U}$ Mann Whitney reveló que los datos obtenidos en cada una de las dimensiones de la GC son significativamente distintos. El grupo empresarial percibe mayores barreras y deficiencias en las capacidades de transferencia de conocimiento universidad - empresa y otras actividades que forman parte de la GC.

PALABRAS CLAVE: Transferencia de conocimiento, sector empresarial, autoridades académicas.

\begin{abstract}
The aim of the study was to evaluate the perception of current knowledge management between universities - companies from the perspective of the academic authorities and executives of Ecuadorian public and private companies. The methodology had a quantitative approach and followed a transversal design, a validated instrument with three dimensions of knowledge management was used: creation, storage and transfer, and application and use of knowledge. The results revealed important differences in the perception of the different activities of the GC between academic authorities and business managers. The U Mann Whitney statistic revealed that the data obtained in each of the dimensions of the GC are significantly different. The business group perceives greater barriers and deficiencies in university-company knowledge transfer capabilities and other activities that are part of the GC.
\end{abstract}

KEYWORDS: Knowledge transfer, business sector, academic authorities. 
INTRODUCCIÓN

La academia posee la capacidad para ofertar al sector empresarial servicios profesionales y así mejorar servicios, modelos de gestión, técnicas de trabajo; permitiendo así que los sectores estratégicos de un país sean competitivos en los nuevos escenarios de competencia local, regional y mundial. Este efecto se puede dar mediante la formación de una red entre académicos y empresarios, lo que permite atender la demanda de innovación en el mercado, reconocer proyectos con potencial en $\mathrm{I}+\mathrm{D}$, y propiciar la colaboración multidisciplinaria (McCaul, 2009). Por estas razones, la discusión sobre la contribución de la universidad, además de la enseñanza y la investigación, en el desarrollo económico a través de la transferencia de conocimientos hacia la industria, ha recibido una gran atención en la literatura sobre gestión estratégica (Anatan, 2013).

Así, actualmente en diversos países a la par con el desarrollo económico, las políticas gubernamentales han comenzado a proveer una plataforma para la colaboración entre universidades, gobierno y la industria (Abbas, Avdic, Xiaobao, Hasan \& Ming, 2019). De hecho, a pesar de que en varias naciones la inserción de las estrategias ligadas a la GC a partir de las instituciones de educación superior aún es incipiente (Castro, Nagano \& Ribeiro, 2019), el creciente interés por la GC muestra que se han establecido muchas políticas para promover la transferencia de conocimiento a través de alianzas entre universidades e industrias basadas en la motivación y los recursos disponibles, con diferentes mecanismos en países de todo el mundo. Uno de estos mecanismos son el tipo de políticas de financiamiento (Abbas et al., 2019), en la mayoría de los países incluidos en la lista de la Organización para la Cooperación y Desarrollo Económino (OCDE), los gobiernos asignan más subvenciones que se centran en la investigación que tienen el objetivo principal de mejorar la estructura institucional y la investigación (Aldrich, 2012).

En Ecuador, este enfoque se empieza a tomar en consideración a partir del 2007, año en el que el gobierno, por medio de la Secretaria Nacional de Educación Superior, Ciencia y Tecnología (SENESCYT), creó la Ley Orgánica de Educación Superior con el objetivo de mejorar la calidad de la educación superior y uno de los puntos que enfatiza la ley es darle impulso a la gestión del conocimiento en las entidades de tercer nivel y de esta manera potenciar el desarrollo del país.
En consecuencia, las universidades del Ecuador tuvieron que realizar cambios en sus estatutos para la incorporación de un Sistema de Gestión del Conocimiento, que permitiera estar acorde a los pedidos de la Ley y poder competir en el rango de la educación y producción del conocimiento a nivel de país.

Dados estos cambios, los cuales han ido configurando nuevas formas de gestión en diversas universidades ecuatorianas, el presente estudio tiene como objetivo evaluar la percepción de la actual gestión del conocimiento y la capacidad de transferencia de conocimiento entre universidad - empresa, desde la perspectiva de las autoridades de una universidad privada, Universidad Católica de Santiago de Guayaquil, y desde la perspectiva de directivos de empresas públicas y privadas ecuatorianas.

\section{REVISIÓN DE LITERATURA}

El campo de la GC puede observarse desde varias perspectivas, entre ellas destacan la filosofía, las ciencias cognitivas, las ciencias sociales, las ciencias de la gestión, las ciencias de la información, la ingeniería del conocimiento, la inteligencia artificial y la economía (Gao, Chai, \& Liu, 2018). En particular, la investigación estratégica como base para la gestión del conocimiento condujo a una teoría de la estrategia basada en el conocimiento y de este enfoque surgieron los conceptos más referidos de la GC como el aprendizaje organizativo, la innovación, el conocimiento tácito y las competencias básicas (Baskerville \& Dulipovici, 2006).

El concepto de GC se origina al observar la importancia de que las organizaciones, de cualquier industria o sector, desarrollen un marco para organizar o estructurar su conocimiento (Gold, Maholtra y Segar, 2001) pues sin ello, no existe consistencia o diálogo común de conocimiento y esto dificulta la gestión eficaz. El conocimiento sobre un tema en particular puede residir en diferentes partes de la organización o en diferentes sistemas dentro de la organización. La combinación o integración de este conocimiento reduce la redundancia, mejora la representación consistente, mejora la eficiencia al eliminar el exceso de volumen de información (Grant, 1996) y permite a la organización reemplazar el conocimiento que se ha quedado obsoleto. El conocimiento diverso debe integrarse para maximizar la eficiencia. Por lo tanto, un objetivo principal de cualquier organización debería ser integrar el conocimiento especializado de $\mathrm{mu}^{-}$ chas áreas o personas (Davenport y Klahr, 1998). 
La GC es vista como un proceso donde se ejecutan muchas actividades relacionadas para llevar a cabo elementos clave de estrategia y operaciones para su gestión eficiente (Gao, Chai \& Liu, 2018), entre estas actividades las más mencionadas en la literatura están la creación, adquisición, almacenamiento, transferencia, aplicación y uso de conocimiento (Tongsami \& Tongsami, 2017; Mahdi, Nassar, \& Almsafir, 2019). Dichas actividades pueden ser vistas como los componentes o dimensiones de la GC, las cuales pueden coincidir entre autores, en particular, Tarí y García (2009) y García (2015) resumen dichas dimensiones en tres componentes reiterados en diversas investigaciones: (a) creación de conocimiento, (b) transferencia y almacenamiento de conocimiento, (c) aplicación y uso del conocimiento.

En particular, la dimensión de transferencia resulta una de las más tratadas por la literatura. La transferencia es el el proceso de difundir o compartir conocimientos (Tongsami \& Tongsami, 2017) y constituye el nivel denominado como cooperación (Bontis \& Serenko, 2009). Su relevancia surge porque entre los principales desafíos de la GC es cómo promover el intercambio de conocimientos con otros. El fin principal de la GC se basa en el intercambio exitoso de conocimientos (Swacha, 2015). De hecho, la idea principal debatida en el campo de la GC está relacionada con la necesidad de un nuevo modelo de transferencia de conocimiento, más eficiente en las condiciones de la nueva economía basada en el conocimiento, donde las universidades se han convertido en organizaciones de transferencia de conocimiento y son responsables de estos procesos (Draghici, Baban, Gogan \& Ivascu, 2015).

La transferencia de conocimiento (TC) se refiere a las múltiples formas en que las empresas y otras organizaciones de investigación pueden explotar el conocimiento de las universidades para generar valor económico y social y para el desarrollo de la industria (OCDE, 2013). Abarca una amplia gama de actividades para apoyar las colaboraciones entre universidades, la industria y el sector público, e incluye una variedad de objetivos, modos y canales (Giuri, Munari, Scandura \& Toschi, 2019).

Uno de los mecanismos para lograr la TC efectiva es la cooperación entre los diferentes actores en una sociedad de conocimiento. Entre los enfoques más extendidos que permite $\mathrm{ex}^{-}$ plicar dicho fenómeno es el conocido concepto del modelo de Triple Hélice, el cual refleja las relaciones entre el gobierno - universidad empresa. El propósito principal de la Triple Hélice es crear una atmósfera adecuada para las relaciones bilaterales y trilaterales, de modo que se pueda compartir el conocimiento entre industrias, instituciones gubernamentales y grupos de investigación académica (Etzkowitz \& Leydesdorff, 2000). El fortalecimiento de dicha relación se puede dar a través de actividades de desarrollo como programas de organizaciones estatales, juntas de asesoría industrial y consejos con la comunidad empresarial; y los resultados se pueden estructurar en tres roles universitarios: educación para producir talento humano, investigación para producir nuevo conocimiento, y transferencia de conocimiento a la sociedad para potenciar el espíritu empresarial, tecnología, y experiencia (Draghici et al., 2015). Durante las fases de colaboración entre la universidad y el gobierno, el rol de los miembros del gobierno es garantizar la implementación de las políticas y los procedimientos estándar del gobierno, donde los miembros de la universidad siguen las pautas dadas para generar conocimiento (Abbas et al., 2019).

Entre otras fundaciones teóricas que pueden explicar el surgimiento de alianzas estratégicas en el contexto de universidad - empresa para la transferencia efectiva de conocimiento, Anatan (2013) cita tres perspectivas: Economía de costos de transacción (ECT), teoría de la firma basada en los recursos (RBT) y teoría de la firma basada en el conocimiento (KBT). En resumen, para la autora, dichas teorías hacen hincapié en explicar a la necesidad de acceso a los recursos como el principal motivo para la formación de alianzas estratégicas, lo que resulta en una ventaja donde dos empresas unen y complementan sus recursos.

En base a estos enfoques, Anatan (2015) construyó su propia perspectiva teórica denominada teoría institucional, la cual explica que las actividades de transferencia de conocimiento de la universidad a la industria son impulsadas por la presión del entorno externo para tener capacidades innovadoras en particular en términos de investigación y desarrollo, así como para mejorar el desempeño organizacional (tanto de la universidad como de la industria) y el desempeño de las alianzas para seguir siendo competitivos y sobrevivir.

Otro enfoque bastante conocido para la explicación de las actividades de transferencia universidad - empresa, fue construido por Nonaka y Takeuchi (2007) en su obra seminal 
The Knowledge-Creating Company. Su obra fue una de las más influyentes pues marca el inicio de una segunda generación en la GC, en la cual el conocimiento adquiere una naturaleza dinámica, ya que se mueve constantemente dentro de la organización entre cuatro procesos: socialización, externalización, combinación e internalización (Abbas et al., 2019); y a partir de esta segunda etapa de la CC, la OCDE produce el estudio de renombre, La Economía Basada en el Conocimiento (Tzortzaki \& Mihiotis, 2014). En su modelo teórico conocido como SECI, Nonaka y Toyama (2003), clasifican al conocimiento en tácito y explícito, y explican que el nuevo conocimiento denominado como tácito proviene de la experiencia directa de las propias organizaciones (tanto universidades como gobiernos) y el conocimiento explícito es aquel que ha sido externalizado y está listo para ser aplicado, después de haber atravesado las distintas fases. La socialización se centra en la vinculación de conocimiento tácito a tácito; la externalización se centra en los procesos de conocimiento tácito a explícito; la combinación se centra en el intercambio de conocimiento explícito a explícito; y la internalización se centra en la transformación de conocimiento explícito a tácito (Nonaka \& Takeuchi, 1995). El modelo SECI, es uno de los fundamentos más usados por muchos investigadores bajo el cual argumentan que el intercambio de conocimiento y la creación de conocimiento son componentes clave de una aplicación exitosa de la GC (Yeh, Yeh \& Chen, 2012; Tzortzaki \& Mihiotis, 2014; Swacha, 2015; Abbas et al., 2019).

MÉTODO

Se elaboró un estudio de enfoque cuantitativo con un diseño transversal y de alcance descriptivo. Para la recolección de datos se utilizó un cuestionario validado por expertos conformado con un total de 22 ítems agrupados en tres dimensiones: (a) creación de conocimiento; (b) almacenamiento y trasferencia de conocimiento; y (c) aplicación y uso del conocimiento, sustentadas en los autores revisados (Tarí \& García, 2009; García, 2015). Los ítems fueron valorados con una escala desde 0 indicando total desacuerdo con la aseveración hasta 3 indicando total acuerdo con la aseveración, como: 0: total desacuerdo, 1: en desacuerdo, y 2: parcialmente de acuerdo, y 3: total acuerdo. Las dimensiones adquisición de información, almacenamiento de conocimiento, transferencia de conocimiento, y empoderamiento estuvieron compuestas por dos reactivos.
Mientras, las dimensiones diseminación de información, interpretación compartida, trabajo en equipo y compromiso con el conocimiento estuvieron compuestas por 3 reactivos.

PARTICIPANTES

La población objetivo correspondió a directivos de empresas y autoridades de la academia en la ciudad de Guayaquil. Se tomaron como muestra aquellos grupos empresariales más grandes de la urbe y las autoridades de una universidad privada.

Por las características de los individuos a encuestar, se realizó un muestreo por conveniencia, el cual es un tipo de muestreo no probabilístico en el que los miembros de la población objetivo cumplen con ciertos criterios prácticos, como fácil acceso, proximidad geográfica, disponibilidad en un momento dado o la voluntad de participar (Etikan, Musa \& Alkassim, 2016). De esta forma, en el estudio se incluyeron un total de 21 autoridades de la institución universitaria y un total de 25 directivos o gerentes del sector empresarial.

PROCESAMIENTO DE DATOS

Los datos fueron procesados en el programa estadístico SPSS v. 22. Se obtuvieron estadísticas descriptivas para los dos grupos (autoridades de universidad y directivos empresariales).

Entre los estadísticos descriptivos se obtuvieron la media y la desviación estándar. Además, se calcularon estadísticos de forma, curtosis y simetría, para evaluar las características de los datos. Posterior a ello, se obtuvieron las puntuaciones para las dimensiones de la GC para cada grupo y se realizó un análisis comparativo en dichos puntajes para determinar si existen diferencias significativas entre los resultados obtenidos en la muestra de autoridades de la academia y directivos empresariales.

El análisis se realizó con el estadístico de U Mann - Whitney, el cual se utiliza como alternativa a una prueba t cuando los datos no se distribuyen normalmente, sin embargo, este estadístico es también una prueba de ubicación y forma (Hart, 2001) por lo que las distribuciones de los puntajes en las dimensiones de la GC fueron graficadas para comparar los grupos.

\section{RESULTADOS}

La Tabla 1 presenta los resultados de los promedios de cada ítem para la muestra de autoridades de la academia y directivos empresariales. En casi todos los ítems, la media obtenida fue 
mayor para el caso de la academia, a excepción del ítem 20 referente a cuán actualizadas son las bases de datos disponibles, en este reactivo el mayor grado de acuerdo fue dado por el sector empresarial.

En particular, la diferencia más alta se dio en el ítem 1, referente a la capacidad de la academia para la recolección de información, en este reactivo las autoridades universitarias obtuvieron un valor muy cercano a 3 , es decir indicaron un total grado de acuerdo, mientras los directivos de la industria no superaron la unidad.

Otra diferencia importante se dio en el ítem 13 referente a la adopción de recomendaciones de la academia por parte del sector empresarial y 16, respecto al establecimiento de acuerdos de cooperación, en los cual las autoridades académicas tuvieron un alto grado de acuerdo respecto a la habilidad de la universidad para acoger recomendaciones del sector empresarial y la facilidad para establecer acuerdos de cooperación con empresas; mientras, los directivos empresariales obtuvieron promedios por debajo de la unidad en estos reactivos indicando un bajo nivel de acuerdo en estos dos aspectos.

Por otro lado, el promedio más bajo se obtuvo en ítems relacionados a transferencia de conocimiento (ítem 5) y a aplicación o uso de conocimiento (ítem 21). En estos ítems la muestra de autoridades académica obtuvo una media inferior a los dos puntos y el sector empresarial registró una media menor a la unidad. Así, se observó que en estos aspectos no se percibe una alta capacidad de transferencia ni aplicación de conocimiento, y esta percepción es aún más baja en el sector empresarial.

La Tabla 2 presenta los niveles promedios para las dimensiones de la GC para ambos grupos. Las autoridades universitarias presentaron un grado de acuerdo, en los reactivos de las dimensiones de la GC, estadísticamente más alto frente a los gerentes empresariales. Esta diferencia se puede ver en los promedios obtenidos en cada una de las dimensiones, donde se verificó además que el promedio más bajo se encuentra en el componente de almacenamiento y trasferencia. Sin embargo, dada la no distribución normal de los datos, se utilizó el estadístico U, el cual lleva a los mismos resultados, donde la mediana y la forma de la distribución de las respuestas entre academia y empresa también fueron significativamente distintas al 99\% de nivel de confianza.

TABLA 1. MEDIA Y DESVIACIÓN ESTÁNDAR DE REACTIVOS DE GC PARA AUTORIDADES UNIVERSITARIAS Y EMPRESA

\begin{tabular}{|c|c|c|c|c|}
\hline \multirow{2}{*}{$\begin{array}{l}\text { REACTIVO } \\
\text { LA ACADEMIA: }\end{array}$} & \multicolumn{2}{|c|}{$\begin{array}{l}\text { AUTORIDADES } \\
\text { UNIVERSITARIAS }\end{array}$} & \multicolumn{2}{|c|}{ EMPRESA } \\
\hline & MEDIA & $\begin{array}{l}\text { DESV. } \\
\text { EST. }\end{array}$ & MEDIA & $\begin{array}{l}\text { DESV. } \\
\text { EST. }\end{array}$ \\
\hline $\begin{array}{l}\text { Recaba información del sector } \\
\text { empresarial }\end{array}$ & 2,92 & 0,28 & 0,85 & 0,88 \\
\hline Realiza estudios de mercado & 1,80 & 0,76 & 0,85 & 0,88 \\
\hline $\begin{array}{l}\text { Comparte información transparente } \\
\text { de manera formal e informal }\end{array}$ & 2,92 & 0,28 & 0,85 & 0,88 \\
\hline $\begin{array}{l}\text { Tiene sistemas de información } \\
\text { permite la compartición del } \\
\text { conocimiento }\end{array}$ & 2,04 & 0,45 & 0,65 & 0,93 \\
\hline $\begin{array}{l}\text { Realiza transferencia de } \\
\text { conocimientos de los productos de } \\
\text { la planta académica }\end{array}$ & 1,20 & 0,71 & 0,50 & 0,69 \\
\hline $\begin{array}{l}\text { Reconoce la importancia de los } \\
\text { nuevos requerimientos de la } \\
\text { sociedad }\end{array}$ & 3,00 & 0,00 & 1,95 & 1,05 \\
\hline $\begin{array}{l}\text { Socializa su sistema de gestión de } \\
\text { conocimiento }\end{array}$ & 1,60 & 0,71 & 0,60 & 0,99 \\
\hline $\begin{array}{l}\text { Implementa procedimientos para } \\
\text { recoger las propuestas del sector } \\
\text { empresarial }\end{array}$ & 2,00 & 0,58 & 1,35 & 0,93 \\
\hline $\begin{array}{l}\text { Tiene bases de datos para } \\
\text { almacenar información }\end{array}$ & 2,00 & 0,41 & 1,30 & 0,80 \\
\hline $\begin{array}{l}\text { Permite acceder a las bases de } \\
\text { datos }\end{array}$ & 2,04 & 0,35 & 0,65 & 0,81 \\
\hline $\begin{array}{l}\text { Para la solución del problema } \\
\text { trabaja con el trípode academia- } \\
\text { empresa-estado }\end{array}$ & 1,56 & 0,87 & 0,45 & 0,60 \\
\hline $\begin{array}{l}\text { Forma equipos para proponer } \\
\text { soluciones innovadores mediante } \\
\text { el diálogo }\end{array}$ & 2,00 & 0,41 & 0,50 & 0,83 \\
\hline $\begin{array}{l}\text { Adopta recomendaciones del sector } \\
\text { empresarial }\end{array}$ & 2,92 & 0,40 & 0,95 & 1,05 \\
\hline $\begin{array}{l}\text { Realiza reuniones donde se informa } \\
\text { a los actores de las novedades }\end{array}$ & 2,16 & 0,47 & 0,70 & 0,92 \\
\hline Establece alianzas y redes externas & 1,88 & 0,53 & 1,25 & 1,16 \\
\hline $\begin{array}{l}\text { Da las facilidades para establecer } \\
\text { acuerdos de cooperación }\end{array}$ & 2,92 & 0,28 & 0,95 & 0,83 \\
\hline $\begin{array}{l}\text { Logra que las sugerencias brindadas } \\
\text { se incorporen en los productos y } \\
\text { servicios }\end{array}$ & 2,88 & 0,44 & 0,75 & 0,85 \\
\hline
\end{tabular}

\begin{tabular}{|c|c|c|c|c|}
\hline $\begin{array}{l}\text { Tiene mecanismos o herramientas } \\
\text { para potenciar el conocimiento entre } \\
\text { empresa-academia }\end{array}$ & 2,04 & 0,45 & 0,70 & 1,03 \\
\hline $\begin{array}{l}\text { Ofrece oportunidades reales } \\
\text { para mejorar las habilidades y } \\
\text { conocimientos de los miembros da } \\
\text { la organización }\end{array}$ & 2,84 & 0,37 & 1,50 & 0,83 \\
\hline Tiene bases de datos actualizadas & 2,04 & 0,45 & 2,80 & 0,52 \\
\hline $\begin{array}{l}\text { Traduce su GC en la generación } \\
\text { de productos y servicios para la } \\
\text { sociedad }\end{array}$ & 1,36 & 0,64 & 0,25 & 0,44 \\
\hline Fomenta la investigación generativa & 1,88 & 0,53 & 0,45 & 0,69 \\
\hline
\end{tabular}


TABLA 2. MEDIA Y DESVIACIÓN ESTÁNDAR DE DIMENSIONES DE GC PARA AUTORIDADES UNIVERSITARIAS Y EMPRESA

\begin{tabular}{|c|c|c|c|c|c|}
\hline \multirow{2}{*}{ DIMENSIÓN } & \multicolumn{2}{|c|}{$\begin{array}{l}\text { AUTORIDADES } \\
\text { UNIVERSITARIAS }\end{array}$} & \multicolumn{2}{|c|}{ EMPRESA } & \multirow{2}{*}{$\begin{array}{l}\text { U MANK- } \\
\text { WHITNEY }\end{array}$} \\
\hline & MEDIA & $\begin{array}{l}\text { DESV. } \\
\text { EST. }\end{array}$ & MEDIA & $\begin{array}{l}\text { DESV. } \\
\text { EST. }\end{array}$ & \\
\hline $\begin{array}{l}\text { Creación de } \\
\text { conocimiento }\end{array}$ & 2,21 & 0,40 & 0,89 & 0,81 & 29 ** \\
\hline $\begin{array}{l}\text { Almacenamiento } \\
\text { y transferencia de } \\
\text { conocimiento }\end{array}$ & 2,04 & 0,35 & 0,85 & 0,54 & $9^{* *}$ \\
\hline $\begin{array}{l}\text { Aplicación y uso de } \\
\text { conocimiento }\end{array}$ & 2,32 & 0,36 & 1,06 & 0,68 & $15^{\star *}$ \\
\hline
\end{tabular}

Nota. ${ }^{* *} \mathrm{p}<.01$, la diferencia en las medianas de los grupos es significativa al $1 \%$
La Figura 1 permite observar la diferencia en la distribución de los puntajes para cada una de las dimensiones de la GC. En la academia, las respuestas se acumulan hacia la cola derecha indicando una frecuencia que se acumula en los rangos de alto grado de acuerdo con los reactivos que refieren a los componentes de la GC. Mientras, la distribución formada a partir de la frecuencia en los puntajes dados por el sector empresarial, se presentan dispersos en todos los rangos, con una prevalencia ligeramente mayor en los rangos de la escala de bajo acuerdo o desacuerdo.

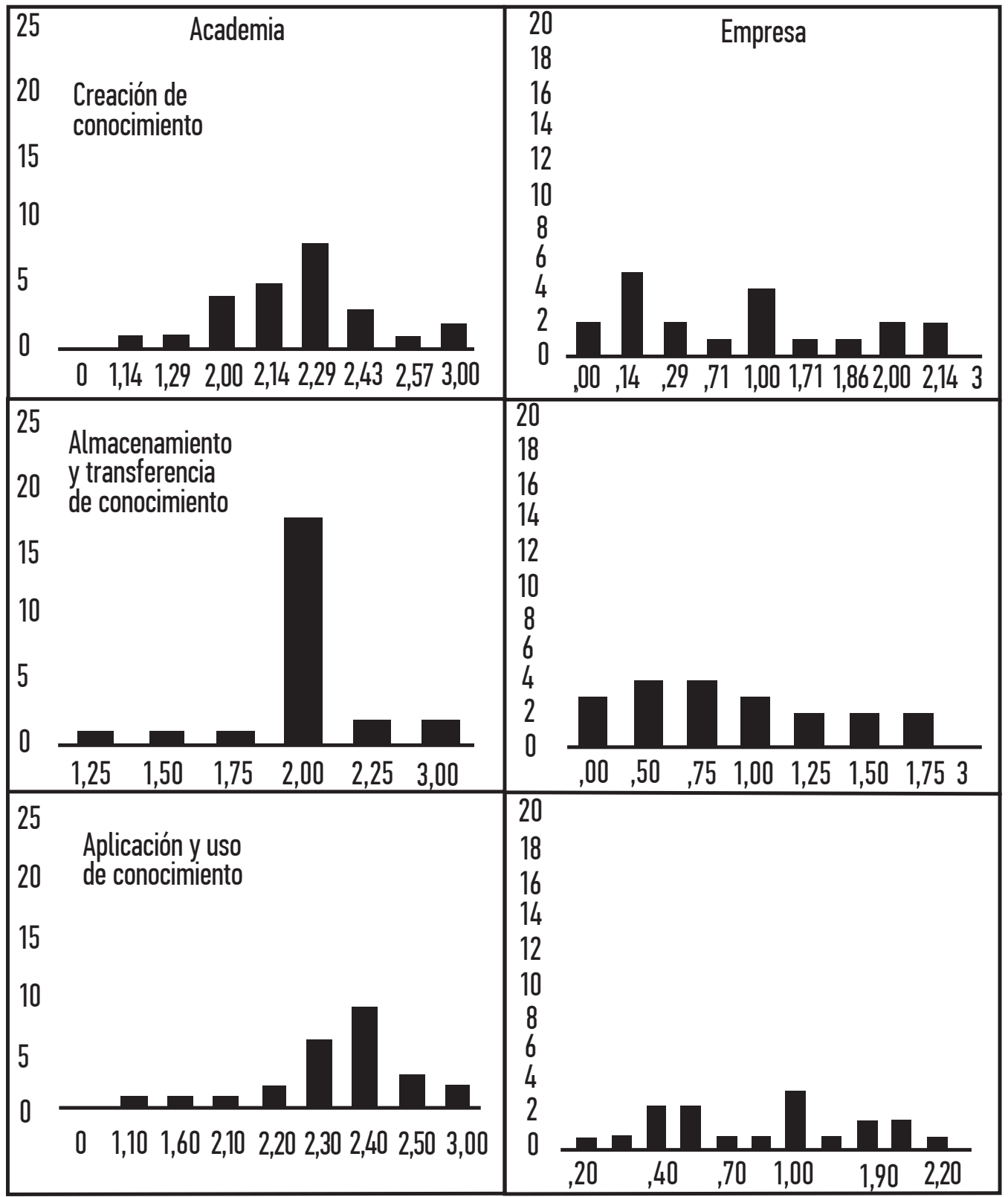

Figura 1 Puntaje para dimensiones de GC para autoridades universitarias y empresa 
DISCUSIONES Y CONCLUSIONES

El estudio permitió la revisión teórica de los conceptos asociados a la gestión del conocimiento y a la fase de transferencia, en la cual interactúan principalmente dos actores empresa y universidad, mientras el gobierno tiene el rol de crear el ambiente propicio para dicha interacción. Partiendo de esta fundamentación teórica se evaluaron los procesos que componen la GC: creación, almacenamiento, transferencia, aplicación y uso del conocimiento; desde dos perspectivas: sector empresarial y academia.

Los resultados obtenidos a través de un análisis concreto con un enfoque cuantitativo, permitieron observar una diferencia significativa en la percepción de la eficiencia de la GC entre las autoridades universitarias y los directivos empresariales. En particular, el sector empresarial obtuvo puntajes más bajos respecto a las diferentes actividades que involucran la CC, sugiriendo que este sector percibe importantes barreras en aspectos como transferencia de conocimiento, recepción de información, formación de alianzas, entre otros.

Dichos resultados, podrían explicarse de distintas formas. En primer lugar, una de las primeras razones que pueden dar origen a esta discrepancia en la GC entre universidad y empresa, es el hecho de que ambas organizaciones tienen características distintitas, aspectos desde su misión hasta su cultura organizacional difieren de manera importante (Anatan, 2015). Incluso, Giuri (2019) manifiesta que las universidades privadas y públicas presentan diferencias entre sí, por lo que las estrategias de la academia, y sobre todo las estrategias denominadas como la Tercera Misión de la universidad que refiere a la compartición de conocimientos, deberían ser estar alienadas a metas y misiones más generales que involucren el desarrollo de la sociedad y ayude a las instituciones de educación superior a posicionarse en el ambiente económico y social actual.

Este estudio refuerza la idea de que con el creciente papel del conocimiento como un recurso organizacional crítico, también crece la importanciadeadministrarloadecuadamente. Incluso se conoce como un factor necesario para la supervivenciaorganizacionalyelmantenimientodela fuerza competitiva". Losfundamentosexpuestos en esta investigación resaltan que la gestión eficaz del conocimiento requiere un intercambio efectivo del conocimiento (Swacha, 2015). Las implicacionesprácticas deestainvestigación son que la universidad, como centro de creación de conocimiento, necesita desarrollar mejor sus oficinasdetransferenciadetecnologíabasadasenla universidad.Estoesesencialparapoderidentificar el valor de cada uno de los nuevos resultados y aportesdelaacademiaenelcontextoempresarial

\section{REFERENCIAS}

Abbas, A., Avdic, A., Xiaobao, P., Hasan, M. M., \& Ming, W. (2019). University-government collaboration for the generation and commercialization of new knowledge for use in industry. Journal of Innovation $\diamond$ Knowledge, 4(1), 23-31.

Anatan, L. (2013). A proposed framework of university to industry knowledge transfer. Review of Integrative Business and Economics Research, 2(2), 304-325.

Anatan, L. (2015). Conceptual issues in university to industry knowledge transfer Studies: a literature review. Procedia-Social and Behavioral Sciences, 211, 711-717.

Aldrich, H. E. (2012). The emergence of entrepreneurship as an academic field: A personal essay on institutional entrepreneurship. Research Policy, 41(7), 1240-1248.

Baskerville, R., \& Dulipovici, A. (2006). The theoretical foundations of knowledge management. Knowledge Management Research \& Practice, 4(2), 83-105.

Bontis, N., \& Serenko, A. (2009). A causal model of human capital antecedents and consequents in the financial services industry. Journal of intellectual capital, 10(1), 53-69.

Castro, I. J. D., Nagano, M. S., \& Ribeiro, S. X. (2019). Elements that influence knowledge sharing in the university-industry-government collaboration: Case studies in Brazil. Revista de Gestão, 26(1), 61-72.

Davenport, T. H., \& Klahr, P. (1998). Managing customer support knowledge. California management review, 40(3), 195-208.

Draghici, A., Baban, C. F., Gogan, M. L., \& Ivas$\mathrm{cu}$, L. V. (2015). A knowledge management approach for the university-industry collaboration in open innovation. Procedia Economics and Finance, 23, 23-32.

Etikan, I., Musa, S. A., \& Alkassim, R. S. (2016). Comparison of convenience sampling and purposive sampling. American journal of theoretical and applied statistics, 5(1), 1-4.

Etzkowitz, H., \& Leydesdorff, L. (2000). The dynamics of innovation: From national systems and "Mode 2" to a triple helix of universityindustry-government relations. Research Policy, 29(2), 109-123. 
Gao, T., Chai, Y., \& Liu, Y. (2018). A review of knowledge management about theoretical conception and designing approaches. International Journal of Crowd Science, 2(1), 42-51.

García, M. (2015). How to measure knowledge management: dimensions and model. Vine, 45(1), 107-125.

Giuri, P., Munari, F., Scandura, A., \& Toschi, L. (2019). The strategic orientation of universities in knowledge transfer activities. Technological Forecasting and Social Change, 138, 261-278

Grant, R. M. (1996). Toward a knowledge based theory of the firm. Strategic management journal, 17(S2), 109-122.

Hart, A. (2001). Mann-Whitney test is not just a test of medians: differences in spread can be important. Bmj, 323(7309), 391-393.

Mahdi, O. R., Nassar, I. A., \& Almsafir, M. K. (2019). Knowledge management processes and sustainable competitive advantage: An empirical examination in private universities. Journal of Business Research, 94, 320-334.

McCaul, B. (2009). Knowledge Transer 2.0 - 6 Ways that KT has to change.... Publicado el 21 de agosto de 2009. En http://brianmccaul.jiscinvolve.org

Nonaka, I., \& Takeuchi, H. (1995). The knowledgecreating company. New York: Oxford University Press.

Nonaka, I., \& Toyama, R. (2003). The knowledgecreating theory revisited: knowledge creation as a synthesizing process. Knowledge Management Research $\Theta$ Practice 1(1), 2-10.
Nonaka, I., \& Takeuchi, H. (2007). The knowledge-creating company. Harvard business review, 85(7/8), 162.

OCDE, (2013). Commercialising public research. In: New Trends and Strategies. Organization for Economic Cooperation and Development, Paris

Swacha, J. (2015). Gamification in knowledge management: motivating for knowledge sharing. Polish Journal of Management Studies, 12.

Tarí, J. \& García, M. (2009). Dimensiones de la gestión del conocimiento y de la gestión de la calidad: una revisión de la literatura. Investigaciones europeas de dirección y economía de la empresa, 15(3), 135-148.

Tongsamsi, K., \& Tongsamsi, I. (2017). Instrument development for assessing knowledge management of quality assurers in Rajabhat universities, Thailand. Kasetsart Journal of Social Sciences, 38(2), 111-116.

Tzortzaki, A. M., \& Mihiotis, A. (2014). A review of knowledge management theory and future directions. Knowledge and Process Management, 21(1), 29-41.

Yeh, Y. C., Yeh, Y. L., \& Chen, Y. H. (2012). From knowledge sharing to knowledge creation: A blended knowledge-management model for improving university students' creativity. Thinking Skills and Creativity, 7(3), 245-257. 